\title{
Parthenolide suppresses non-small cell lung cancer GLC-82 cells growth via B-Raf/MAPK/Erk pathway
}

\author{
Minting Lin ${ }^{1, *}$, Hong $\mathrm{Bi}^{2, *}$, Yanyan Yan ${ }^{3, *}$, Wenjing Huang ${ }^{1}$, Guiping Zhang ${ }^{1}$, Genshui \\ Zhang ${ }^{1}$, Sili Tang ${ }^{1}$, Yun Liu ${ }^{1}$, Lingling Zhang ${ }^{4}$, Jinxiang Ma ${ }^{5}$, Jianye Zhang ${ }^{1}$ \\ ${ }^{1}$ School of Pharmaceutical Sciences and The Fifth Affiliated Hospital, Guangzhou Medical University, Guangzhou 511436, \\ People's Republic of China \\ ${ }^{2}$ Department of Pathology, Shanxi Provincial People's Hospital, Taiyuan 030012, People's Republic of China \\ ${ }^{3}$ Institute of Respiratory and Occupational Diseases, Collaborative Innovation Center for Cancer, Medical College, Shanxi \\ Datong University, Datong 037009, People's Republic of China \\ ${ }^{4}$ Pharmaceutical Department, The Fifth Affiliated Hospital of Guangzhou Medical University, Guangzhou 510700, People's \\ Republic of China \\ ${ }^{5}$ School of Public Health, Guangzhou Medical University, Guangzhou 511436, People's Republic of China \\ *These authors have contributed equally to this work
}

Correspondence to: Jianye Zhang, email: jianyez@163.com

Keywords: non-small cell lung cancer, parthenolide, B-Raf, MAPK/Erk pathway, c-Myc

Received: December 22, 2016

Accepted: February 12, 2017

Published: February 21, 2017

\section{ABSTRACT}

Non-small cell lung cancer (NSCLC), one type of lung cancer, owns high rates of morbidity and mortality. B-Raf is one of the promising oncogenic drivers of NSCLC. Parthenolide, a natural product, is mainly extracted from the herbal plant Tanacetum parthenium. The effect of parthenolide on NSCLC cells and its potential as B-Raf inhibitor were studied in this study. It's shown that parthenolide exhibited the strong cytotoxicity against NSCLC cells with IC50 ranging from $6.07 \pm 0.45$ to $15.38 \pm$ $1.13 \mu \mathrm{M}$. Parthenolide was also able to induce apoptosis, suppress proliferation and invasion in NSCLC cells. In terms of the involved mechanism, parthenolide suppressed GLC-82 cell response via targeting on B-Raf and inhibiting MAPK/Erk pathway signaling. The effect of parthenolide on B-Raf and MAPK/Erk pathway was further confirmed by RNA interference of B-Raf. Decreased expression of C-Myc in protein and mRNA level was also discovered, which is considered as the further downstream of the MAPK/Erk pathway. In addition, STAT3 activity inhibition by parthenolide contributed to its effect on GLC-82 cells, which is independent of PI3K pathway signaling and GSK3. All above provide an insight to understand the action of parthenolide as a potential B-Raf inhibitor in treatment of NSCLC.

\section{INTRODUCTION}

Based on WHO estimates, cancer now causes more death than all coronary heart disease or all stroke [1]. Lung cancer, among different kinds of cancers, remains the most common cancer in the world, both in term of new cases (1.8 million cases, $12.9 \%$ of total) and death (1.6 million deaths, $19.4 \%$ ) because of the high case fatality [2, 3]. It is usually divided into two categories, non-small cell lung cancer (NSCLC) and small cell lung cancer (SCLC), of which the former accounts for about $80 \%$. Surgery, radiotherapy, chemotherapy, molecular targeted therapy and combined treatment of them are the common treatments for NSCLC. However, most of NSCLC patients were locally advanced and advanced at their first visit, with five years survival rates lower than $10 \%$ and $5 \%$ [4]. Drug therapy, including chemotherapy and molecular-targeted therapy, is the main treatment for this staging and plays an important role in it.

Thoracic oncology has witnessed an unprecedented outburst of knowledge regarding molecular biology of NSCLC during the last decade. A number of oncogenic drivers, such as $E G F R, A L K, K R A S, B-R A F$, have emerged as novel molecular targets with potential therapeutic implications [5, 6]. Molecular targeted therapy, due to its 
high efficiency and low toxicity, has received more and more attention [7].

Natural products from Chinese herbal medicine are important source of anti-cancer drug development. It's reported that nearly $67 \%$ of the anti-cancer drugs are natural products or natural products derivatives and that more than 200 kinds of them are now in preclinical or clinical trials $[8,9]$.

Parthenolide (Figure 2A) as a natural sesquiterpene lactone, is mainly extracted from the herbal plant Tanacetum parthenium and has exhibited anti-tumor activities on various tumors including lung, leukemia, pancreatic and breast cancer [10]. Though its effect on lung cancer has ever been reported, its influence on NSCLC and oncogenic drivers of NSCLC was little known. This study was designed to further investigate the cytotoxicity of parthenolide against NSCLC cells and illustrate its potential as B-Raf inhibitor, which is a promising therapeutic strategy for NSCLC. The relative mechanism was also discussed in this study.

\section{RESULTS}

\section{B-Raf and c-Myc were highly expressed in human NSCLC tissues}

Expression of B-Raf and c-Myc (common mutated gene in many cancers) in NSCLC were investigated by immunohistochemistry (IHC) analysis. Figure 1 showed the positive and negative expression of them. B-Raf was highly expressed in 33 out of 50 cases with positive expression rate of $88.0 \%$, which suggested that B-Raf is a promising oncogenic driver for molecular-targeted therapy. High Expression of c-Myc was also found in human NSCLC tissues with positive expression rate of $76.0 \%$. Statistical analysis results based on age, gender, histological grade and stage were summarized in Table 1.

\section{Parthenolide and other sesquiterpene lactones showed potent cytotoxicity against human NSCLC cells}

MTT assays were carried out with a variety of human lung cancer cells to test the activity of parthenolide and other sesquiterpene lactones. Human lung cancer cells consisted of five NSCLC cell lines, GLC-82, A549, H1650, H1299 and PC-9 cells. As results showed in Figure 2B-2F, parthenolide exhibited potent cytotoxicity towards GLC-82, A549, PC-9, H1650 and H1299 cells, with IC50 values of $6.07 \pm 0.45,15.38 \pm 1.13,15.36 \pm 4.35,9.88$ \pm 0.09 and $12.37 \pm 1.21 \mu \mathrm{M}$, respectively. Among them, parthenolide showed the strongest activity against GLC82 cells. Therefore, GLC-82 cells were chosen for further research. Cell status before and after parthenolide treatment was revealed in Figure 2G. Dabrafenib as positive control and other sesquiterpene lactones were also investigated to elucidate their IC50 values against NSCLC cell lines, which were listed in Table 2. Parthenolide, with the strongest potential, was thus selected for further research with GLC-82 cells.

\section{Parthenolide inhibited migration, proliferation in GLC-82 Cells}

As mentioned above, parthenolide exerted potent inhibition on cell growth in different lung cancer cells, especially GLC-82 cells. To further demonstrate its effect on migration and proliferation, scratch wound healing assay and clone formation assay were carried out. Results revealed that parthenolide inhibited wound healing of the cells in time and dose-dependent manners (Figure 3A, 3B) and suppressed clone formation time-dependently (Figure 3C). It's suggested that parthenolide could inhibit human NSCLC cell line GLC-82 migration, proliferation on the basis of its cytotoxicity.

\section{Parthenolide induced apoptosis in GLC-82 cells in dose-dependent manners}

To further confirm whether parthenolide took effects by inducing apoptosis, Annexin V-FITC/PI double staining was carried out. As shown in Figure 4, parthenolide induced GLC-82 cells apoptosis along with the increase of drug concentration. The apoptosis rates of control, 5.0, 10.0 and $20.0 \mu \mathrm{M}$ parthenolide against GLC-82 cells were $8.21 \pm 0.21 \%, 19.82 \pm 0.62 \%, 27.17 \pm 1.20 \%$ and $37.30 \pm 2.41 \%$, respectively.

\section{Parthenolide downregulated the expression of B-Raf, c-Myc and phosphorylation of MEK, Erk in GLC-82 cells}

To investigate the potential of parthenolide as B-Raf inhibitor, western blot and RT-QPCR were applied for detection. When GLC-82 cells were treated with $20.0 \mu \mathrm{M}$ parthenolide for $0-48 \mathrm{~h}$, the expression of B-Raf in protein (Figure 5A) and mRNA (Figure 5C) level decreased in turn. Expression of c-Myc was also measured in the same way. When GLC-82 cells were treated with the longer medication time, protein and mRNA level of c-Myc were lower (Figure 5B, 5D). What's more, phosphorylation of MEK and Erk was suppressed after exposure to different concentration of parthenolide for $6 \mathrm{~h}$, while the total protein level of MEK and Erk didn't change (Figure 5E).

\section{Parthenolide suppressed MAPK/Erk pathway signaling in GLC-82 Cells}

It was found above that parthenolide could inhibit the expression of B-Raf and c-Myc. To further study the interaction between them and their effect on MAPK/Erk pathway, siRNA-interference technique was employed. The results exhibited that the expression of c-Myc, p-MEK and p-Erk were downregulated after the transfection of 
Table 1: B-Raf and c-Myc expression with clinicopathological variables in 50 NSCLC samples

\begin{tabular}{|c|c|c|c|c|c|c|}
\hline \multicolumn{2}{|c|}{ Clinicopathological parameters } & \multirow{2}{*}{$\frac{n}{29}$} & \multirow{2}{*}{$\frac{\text { B-Raf }}{25}$} & \multirow{2}{*}{$\frac{\boldsymbol{P}}{0.986^{\#}}$} & \multirow{2}{*}{$\frac{\text { c-Мyc }}{24}$} & \multirow{2}{*}{$\frac{\boldsymbol{P}}{0.189}$} \\
\hline Age(years) & $\geq 60$ & & & & & \\
\hline & $<60$ & 21 & 19 & & 14 & \\
\hline \multirow[t]{2}{*}{ Gender } & Male & 33 & 30 & $0.673^{\#}$ & 25 & $1.000^{\#}$ \\
\hline & Female & 17 & 14 & & 13 & \\
\hline \multirow[t]{3}{*}{ Histological Grade } & Well-differentiate & 3 & 3 & $1.000^{*}$ & 1 & $0.179^{*}$ \\
\hline & Moderately-differentiate & 27 & 24 & & 22 & \\
\hline & Poor-differentiate & 20 & 17 & & 15 & \\
\hline \multirow[t]{4}{*}{ Stage } & 0 & 1 & 1 & $0.244^{*}$ & 0 & $0.292^{*}$ \\
\hline & $\mathrm{I}$ & 23 & 22 & & 19 & \\
\hline & II & 22 & 17 & & 16 & \\
\hline & III & 4 & 4 & & 3 & \\
\hline
\end{tabular}

\#continuity correction Chi-square *Fisher's Exact Test.

Table 2: Sesquiterpene lactones and Dabrafenib cytotoxicity to NSCLC cell lines

\begin{tabular}{lccccc}
\hline \multirow{2}{*}{ Compound } & \multicolumn{5}{c}{ IC50 $(\boldsymbol{\mu M})$} \\
\cline { 2 - 6 } & GLC-82 & A549 & H1650 & PC-9 & H1299 \\
\hline Dehydrocostus lactone & $12.36 \pm 1.89$ & $24.40 \pm 1.38$ & $30.79 \pm 1.20$ & $14.30 \pm 1.49$ & $15.34 \pm 1.17$ \\
Epoxymicheliolide & $11.13 \pm 0.35$ & $24.51 \pm 4.94$ & $24.23 \pm 3.84$ & $32.91 \pm 2.97$ & $28.44 \pm 1.07$ \\
Micheliolide & $11.32 \pm 2.59$ & $22.90 \pm 1.33$ & $19.81 \pm 0.92$ & $22.82 \pm 0.89$ & $19.03 \pm 1.81$ \\
Arglabin & $6.66 \pm 0.04$ & $17.09 \pm 1.91$ & $21.48 \pm 4.14$ & $19.96 \pm 0.55$ & $20.22 \pm 1.61$ \\
Isoalantolactone & $17.09 \pm 1.68$ & $17.12 \pm 1.62$ & ND & ND & ND \\
Alantolactone & $35.99 \pm 5.55$ & $32.89 \pm 5.60$ & ND & ND & ND \\
Dabrafenib (IC50 nM) & $6.12 \pm 0.98$ & $5.49 \pm 0.77$ & $1.45 \pm 0.24$ & $2.19 \pm 0.26$ & $1.42 \pm 0.33$ \\
\hline
\end{tabular}

ND: not detected.

B-Raf siRNA and further downregulation was found when combined with parthenolide (Figure 6A, 6C). On the other hand, levels of B-Raf, p-MEK and p-Erk showed no changes after the transfection of c-Myc siRNA (Figure 6D, 6F). They were further confirmed in mRNA level by RT-QPCR, as shown in Figure 6B, 6E. It's speculated that parthenolide targeted on B-Raf and inhibited the MAPK/Erk pathway signaling. C-Myc was probably the further downstream of MAPK/Erk pathway, which had no feedback on the B-Raf expression and MAPK/Erk signaling.

\section{Parthenolide decreased STAT3 activity and had no changes in GSK3 $\alpha / \beta$ and Akt}

Other than the above findings, parthenolide was also found to inhibit the phosphorylation of STAT3, which may partly attribute to the potent activity of parthenolide. After treatment with 2.5, 5.0, 10.0, 20.0 $\mu \mathrm{M}$ parthenolide, p-STAT3 level expressed as gray value was
$99.47 \pm 4.90,84.98 \pm 2.16,45.86 \pm 2.18,13.40 \pm 0.71 \%$ of control, respectively (Figure 7A, 7B). On the other hand, parthenolide had no influence on both total and phosphorylated level of Akt and GSK $3 \alpha / \beta$. After exposure to parthenolide of $2.5,5.0,10.0,20.0 \mu \mathrm{M}$ parthenolide, p-GSK3 $\beta$ level was $99.79 \pm 6.86,100.58 \pm 10.05$, $95.80 \pm 4.05,103.79 \pm 7.17 \%$ of control, respectively (Figure 7A, 7C). After GLC-82 cells were treated with 2.5, 5.0, 10.0, $20.0 \mu \mathrm{M}$ parthenolide, p-AKT level was $100.32 \pm 5.35,100.88 \pm 1.22,98.74 \pm 3.68,100.61 \pm 8.83 \%$ of control, respectively (Figure 7A, 7D).

\section{DISCUSSION}

B-Raf, short for $\mathrm{v}$-Raf murine sarcoma viral oncogene homolog $\mathrm{B}$, is a member of serine/threoninespecific protein kinases [11, 12]. Different types and frequency of B-Raf mutations associated with human cancers have been identified [13-16]. Notably, B-Raf was 
also considered as one of the oncogenic drivers for NSCLC with frequency of about 3\% [17-19]. Differentially high expression of B-Raf in human NSCLC tissues was confirmed by immunohistochemistry in our study too, with positive expression rate of $88.0 \%$ (Figure 1). The rate in our IHC analysis was higher than that of the published records and this is probably because the NSCLC tissues we examined are all lung adenocarcinoma tissues which were reported to have a higher frequency of B-Raf mutation. Our results showed that B-raf or c-Myc positive rates exhibited no difference when age, gender, histological grade and clinical stage were concerned. It's unknown whether the difference will be significant when the number of clinical samples is more than 50 .

Drugs that treat cancer driven by B-Raf mutations have been developed, such as Vemurafenib and Dabrafenib. These two drugs are approved by FDA for treatment of late-stage melanoma and B-Raf-mutant lung adenocarcinomas. It's proved that B-Raf inhibitors improved rates of overall and progression-free survival in patients and showed potent potential in treatment of B-Raf-mutant cancers [20-22]. In this term, B-Raf inhibitors were the potential strategy for NSCLC treatment. The active compound of feverfew (Tanacetum parthenium), parthenolide, has shown potential significant cancer suppression activity in vitro and in vivo. Although the effect of parthenolide as an inhibitor of $\mathrm{NF}-\kappa \mathrm{B}$ activity has been reported previously in several cancers, its significance, detailed mechanism as B-Raf inhibitor for NSCLC has not yet been investigated [23, 24].

MTT assays, in this way, were firstly carried out to test the cytotoxicity of parthenolide to different human
NSCLC cells. In consistence to its effect on other cancer types, parthenolide also exhibited potent inhibition to NSCLC cells, especially GLC-82 cells (Figure 2). Furthermore, parthenolide could inhibit human NSCLC cell line GLC-82 migration, proliferation and induce apoptosis on the basis of its cytotoxicity (Figures 3, 4).

Members of the Raf family encode serine/threonine protein kinases and play a role in regulating the MAP kinase (MAPK)/ ERKs signaling pathway through direct interaction and phosphorylation. The canonical MAPK/ Erk pathway is composed of three types of MAPKKK: A-Raf, B-Raf and C-Raf kinases. B-Raf is the gene most commonly mutated at this level in human cancer and shown to display higher MEK kinase activity than other members [25]. Western blot and RT-QPCR results showed that parthenolide suppressed expression of B-Raf in both protein and mRNA levels (Figure 5A, 5C). Combined with the IHC results (Figure 1), it's preliminarily proposed that parthenolide is able to target on B-Raf mutation and then inhibit the development of NSCLC including proliferation and invasion.

Levels below B-Raf of the MAPK cascade are MAPKKs, which are composed of MEK1 and MEK2. Erk1 and Erk2 are the further downstream and the final effectors of the MAPK pathway [26]. As shown in Figure 5E, phosphorylation of MEK and Erk was decreased after exposure to different concentration of parthenolide for $6 \mathrm{~h}$, while the total protein level of MEK and Erk didn't change. Erk phosphorylation can lead to activation of multiple substrates that are responsible for stimulation of cell proliferation. Spatial localization of ERK determines target substrates and later effects within the cell [27]. When
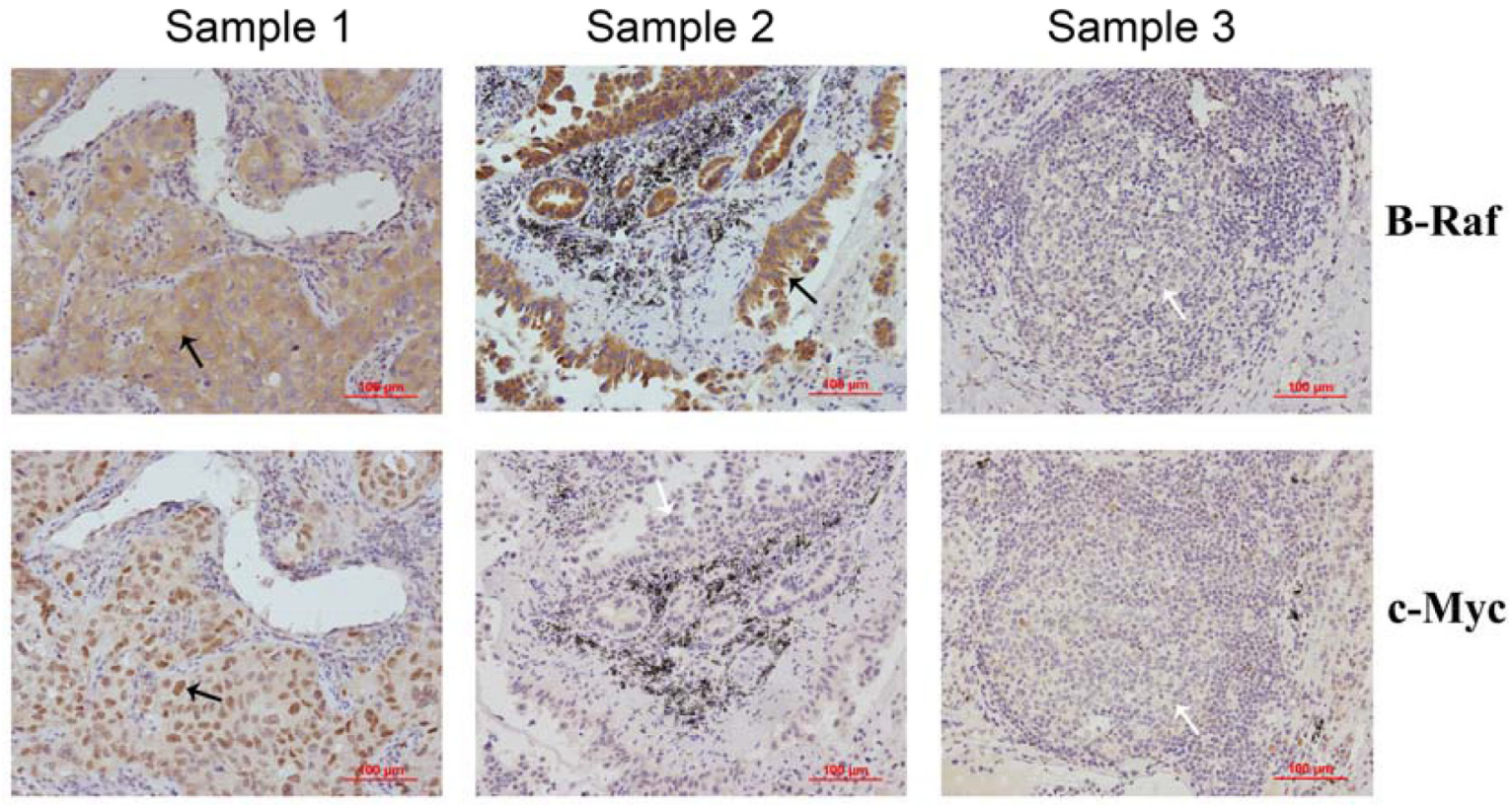

Figure 1: Expression of B-Raf and c-Myc proteins in human NSCLC samples. The level of B-Raf and c-Myc in 50 human lung adenocarcinoma samples were examined by immunohistochemistry with specific antibodies. Three representative samples are shown, where either B-Raf or c-Myc staining were negative (white arrow) and positive (black arrow). 
located at nucleus, active Erk causes phosphorylation and activation of various transcription factors such as c-Fos, c-Jun, Elk-1, c-Myc and ATF2 promoting cell progression [28]. For one thing, as GLC-82 cells were treated with the longer medication time, protein and mRNA level of c-Myc were lower in our study (Figure 5B, 5D). For another thing, parthenolide had no influence on both total and phosphorylated level of GSK $3 \alpha / \beta$ (Figure 7). These suggested that parthenolide might inhibit Erk to locate at nucleus and thereby downregulate the expression of c-Myc in human NSCLC GLC-82 cells.

To illustrate whether parthenolide specifically target on B-Raf and the relationship between B-Raf and c-Myc, siRNA interference was taken. Results (Figure 6) indicated that parthenolide specifically targeted on B-Raf and then inhibited the MAPK/Erk pathway signaling. c-Myc, on the other hand, was probably the further downstream of MAPK/Erk pathway, which had no feedback on the B-Raf expression and MAPK/Erk signaling. However, further research about the interaction between them are still in need and will be taken next.

STAT proteins are extracellular ligand-responsive transcription factors that mediate broadly diverse biological processes, including cell proliferation, transformation, apoptosis and differentiation. STAT protein, especially STAT3, are usually phosphorylated aberrantly in tumors, inducing malignant proliferation and apoptosis inhibition [29]. It's reported that parthenolide can inhibit STAT3 activity and therefore suppress the development of tumor [30]. Parthenolide, in our study,

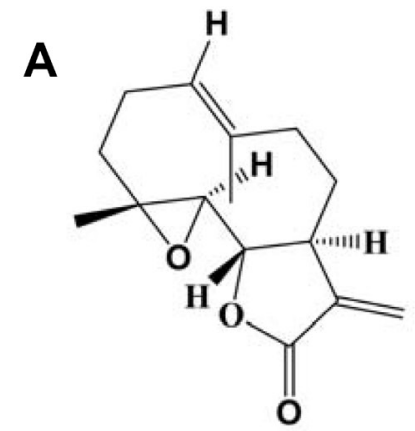

Parthenolide

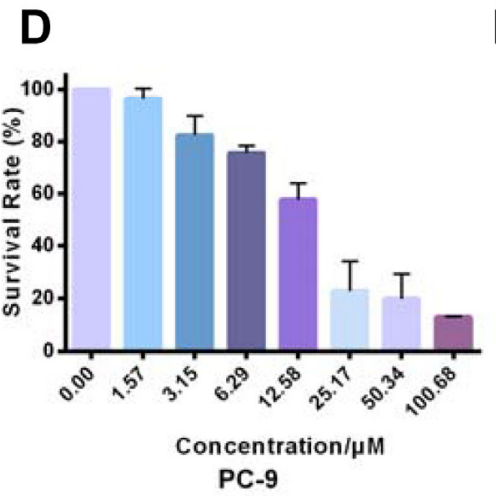

G

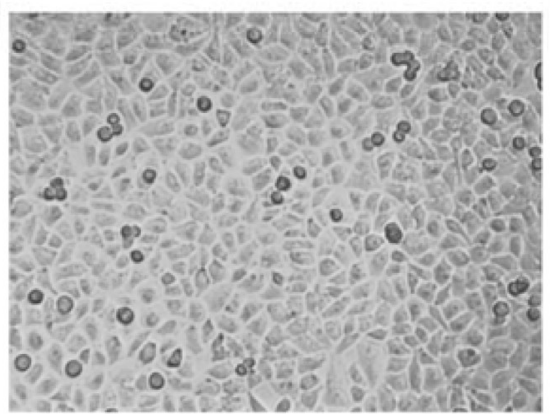

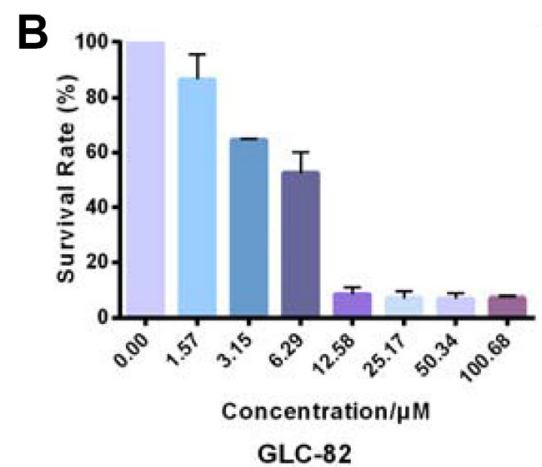

E

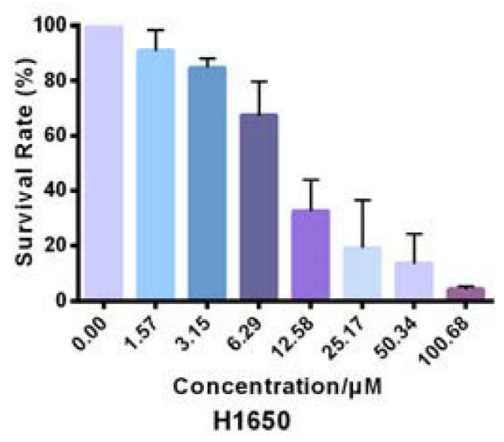

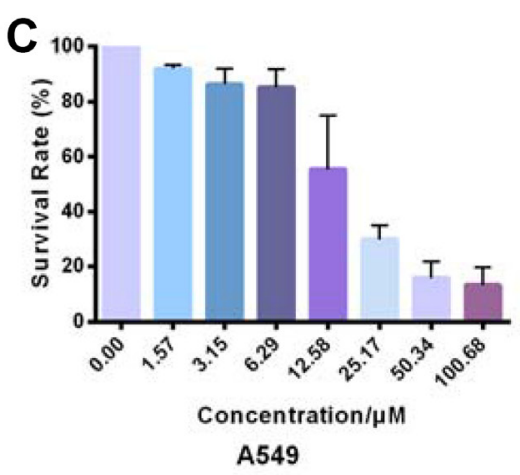

F

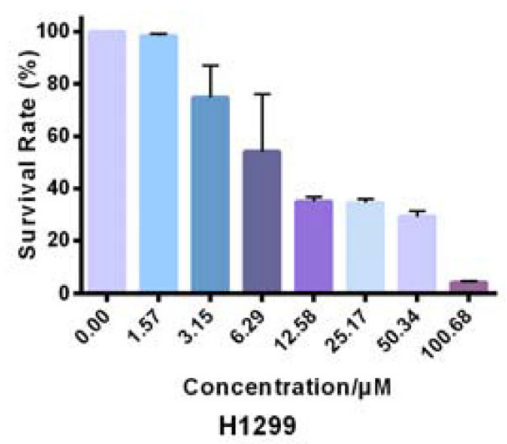

$10 \mu \mathrm{M} / 72 \mathrm{~h}$

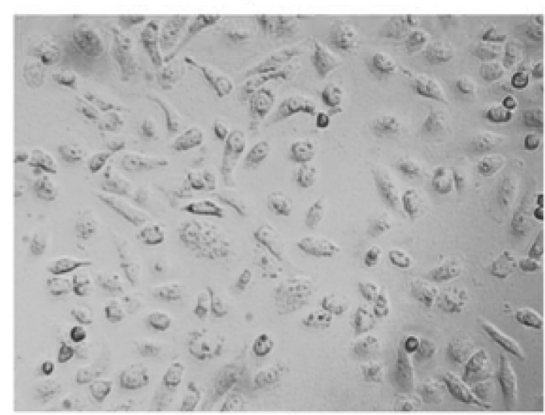

Figure 2: Chemical structure of parthenolide and its effect on different NSCLC cells. (A) Chemical structure of parthenolide (molecular weight: $248.32 \mathrm{~g} / \mathrm{mol}$ ); (B-F) Parthenolide inhibited GLC-82, A549, PC-9, H1650 and H1299 cells survival. Survival (\%) $=($ mean experimental absorbance/mean control absorbance $) \times 100 \% ;(\mathbf{G})$ GLC-82 cell status before and after parthenolide treatment. 


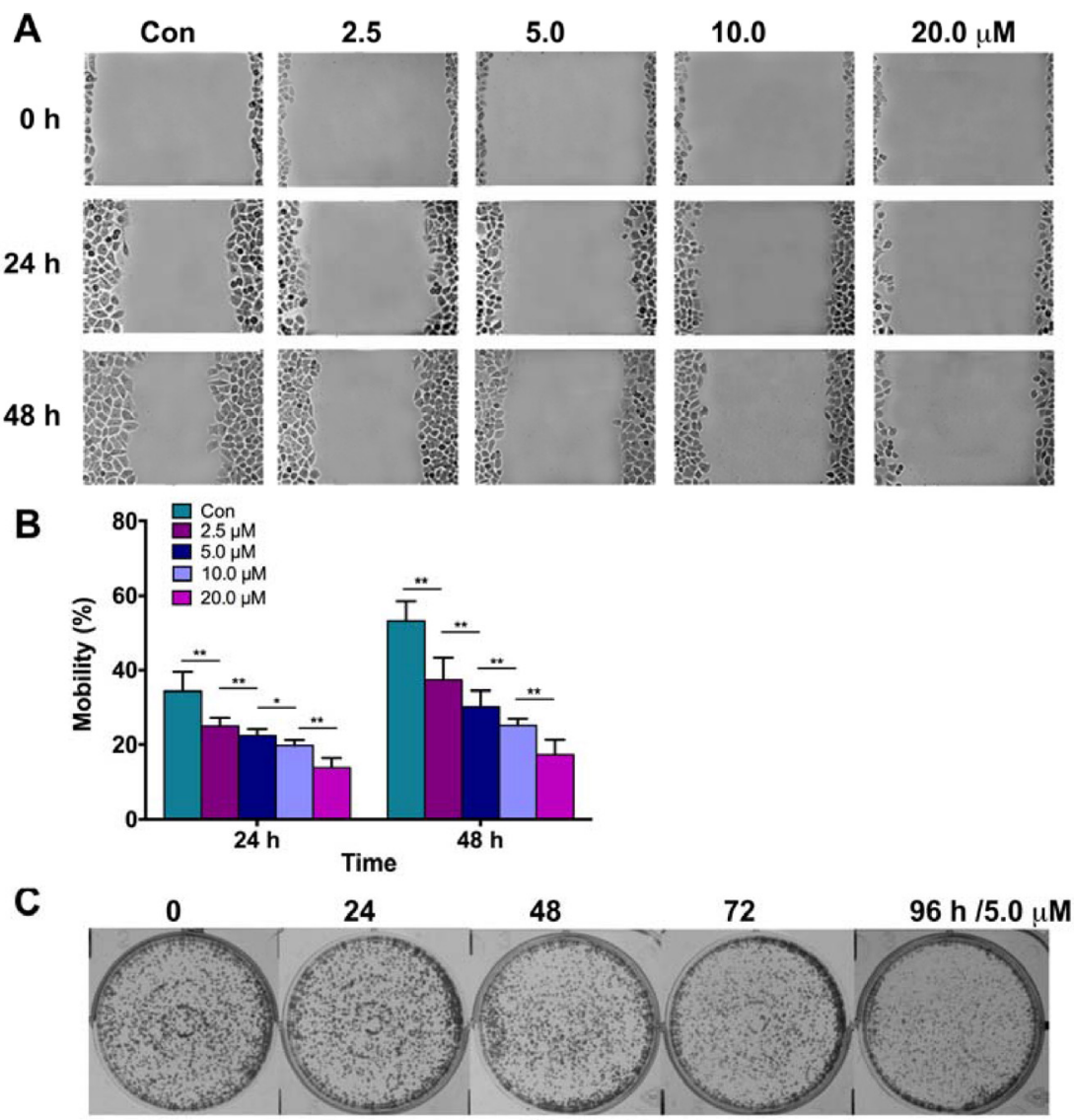

Figure 3: Effects of parthenolide on migration and colon formation of NSCLC cells. (A) GLC-82 cells migration was inhibited by parthenolide in dose-dependent manner; (B) Data analysis of (A). (C) Parthenolide reduced colon formation of GLC-82 cells. NS $=$ no significance, ${ }^{*} p<0.05, * * p<0.01, n \geq 3$.

A

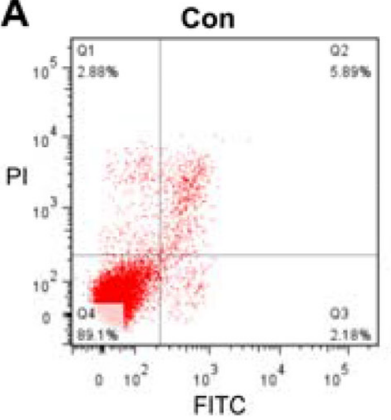

5.0
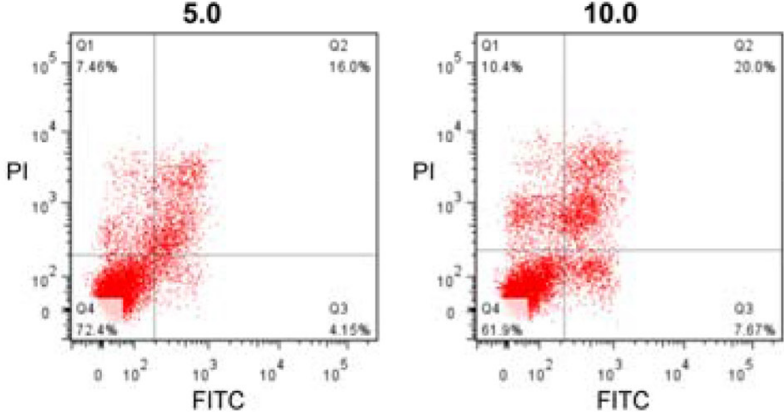

$20.0 \mu \mathrm{M} / 72 \mathrm{~h}$

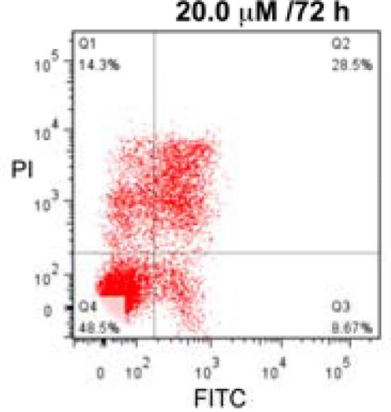

B

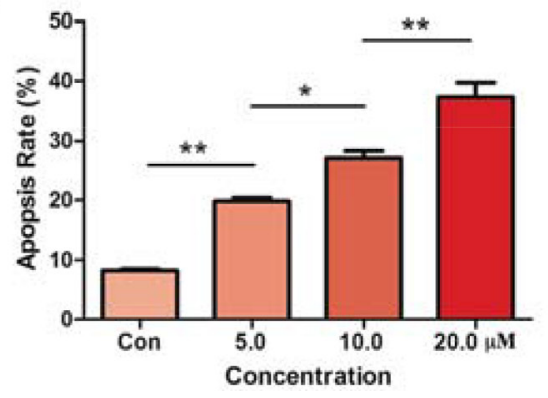

Figure 4: Effects of parthenolide on apoptosis of NSCLC cells. (A) Parthenolide concentration-dependently induced GLC-82 cells apoptosis, detected by Annexin V-FITC/PI double staining; (B) Data analysis of (A). ${ }^{*} p<0.05,{ }^{* *} p<0.01, n \geq 3$. 


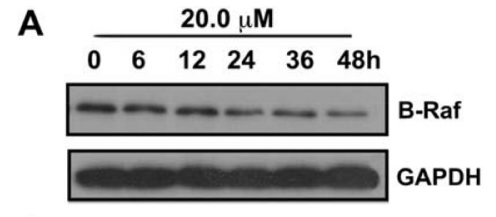

C

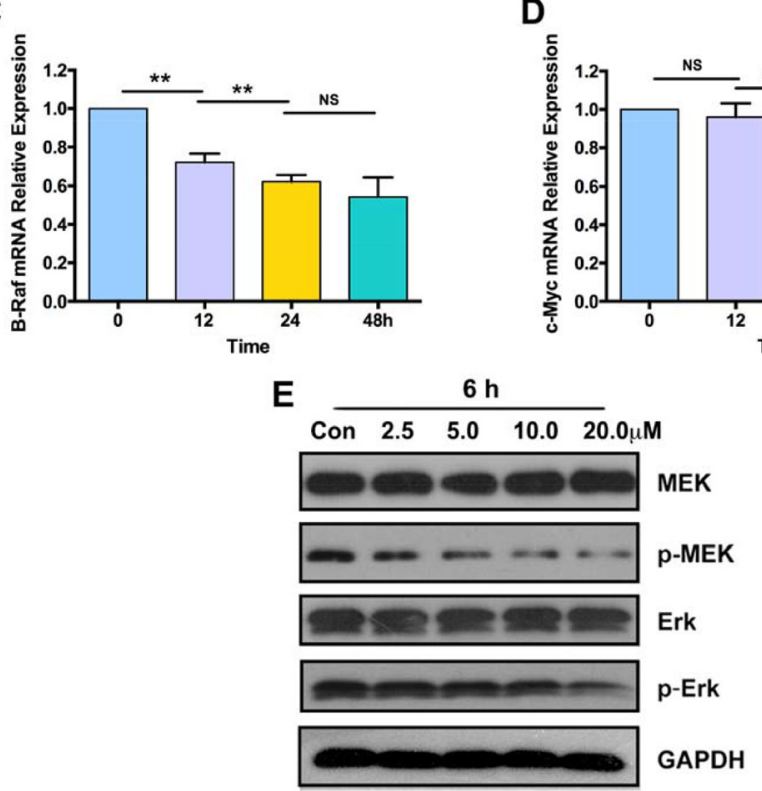

B

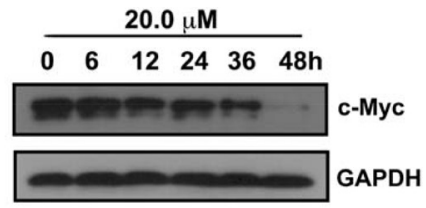

D

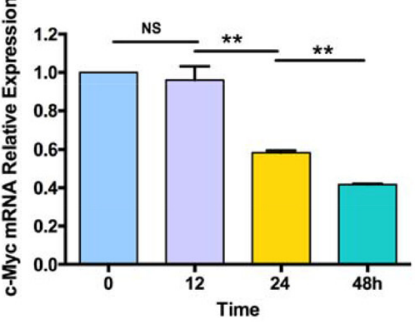

Figure 5: Parthenolide suppressed expression of B-Raf, c-Myc and phosphorylation of MEK, Erk. (A, B) Protein expression of B-Raf and c-Myc after treatment with $20.0 \mu \mathrm{M}$ parthenolide for 0, 6, 12, 24, 36 and $48 \mathrm{~h}$; (C, D) B-Raf and c-Myc mRNA levels detected by RT-QPCR after exposure of $20.0 \mu \mathrm{M}$ parthenolide for indicated time; (E) Level of MEK, p-MEK, Erk, p-Erk detected after treatment with $0,2.5,5.0,10.0,20.0 \mu \mathrm{M}$ parthenolide for $6 \mathrm{~h}$. NS $=$ no significance, ${ }^{*} p<0.05,{ }^{* *} p<0.01, n \geq 3$.
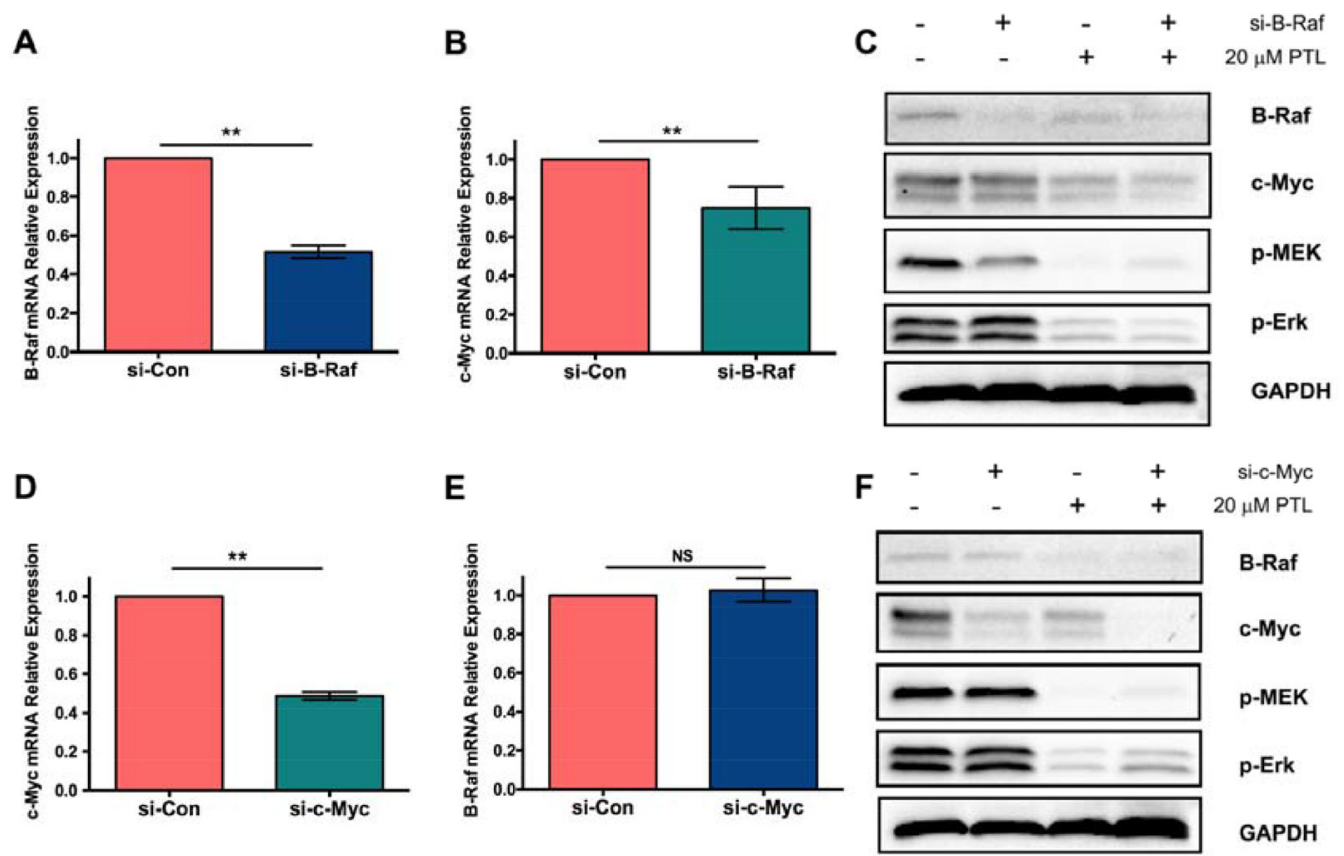

Figure 6: Effect of parthenolide on MAPK/Erk pathway signaling and c-Myc expression. (A) Interference confirmed by RTQPCR after si-B-Raf transfection; (B) c-Myc mRNA expression after transfection with si-B-Raf; (C) Level of different proteins detected by western blot after treatment with si-B-Raf and/or parthenolide; (D) Interference confirmed by RT-QPCR after si-c-Myc transfection; (E) B-Raf mRNA expression after transfection with si-c-Myc; (F) Level of different proteins detected by western blot after treatment with si-c-Myc and/or parthenolide. NS $=$ no significance, ${ }^{*} p<0.05, * * p<0.01, n \geq 3$. 
was found to inhibit the phosphorylation of STAT3 (Figure 7), which probably attribute to the potent activity of parthenolide. Deregulation of $\mathrm{PI} 3 \mathrm{~K} /$ protein kinase $\mathrm{B}$ (PKB/AKT)/mammalian target of rapamycin (mTOR) (PI3K) pathway and MAPK pathway frequently occurs in human cancers. Several studies demonstrated that blockade of one pathway may lead to the activation of the other signaling cascade [31]. Akt, as the critical factor of PI3K pathway, was examined in our research too. Interestingly, parthenolide had no influence on both total and phosphorylated level of Akt, which indicated that the effect of parthenolide on MAPK pathway didn't activate the PI3K pathway signaling. The potent cytotoxicity of parthenolide may be also explained in this way.

\section{MATERIALS AND METHODS}

\section{General experimental procedures}

Parthenolide (PTL), Dehydrocostus, Epoxymicheliolide, Micheliolide, Arglabin, Isoalantolactone and Alantolactone with purity of HPLC $\geq 98 \%$ were purchased from Nanjing Spring \& Autumn Biological Engineering Co., Ltd (Nanjing, China). Dabrafenib was purchased from MCE Co.,Ltd.(Monmouth Junction, NJ). RPMI 1640, DMEM and Opti-MEM $(1 \times)$ medium (Gibco, Carlsbad, CA), Block it $^{\mathrm{TM}}$ Alexa Fluor ${ }^{\circledR}$ Red Fluorescence Control and Lipofectamine ${ }^{\circledR} 2000$ (Invitrogen, Carlsbad, CA), TRIzol ${ }^{\circledR}$ Reagent (Ambion, Carlsbad,
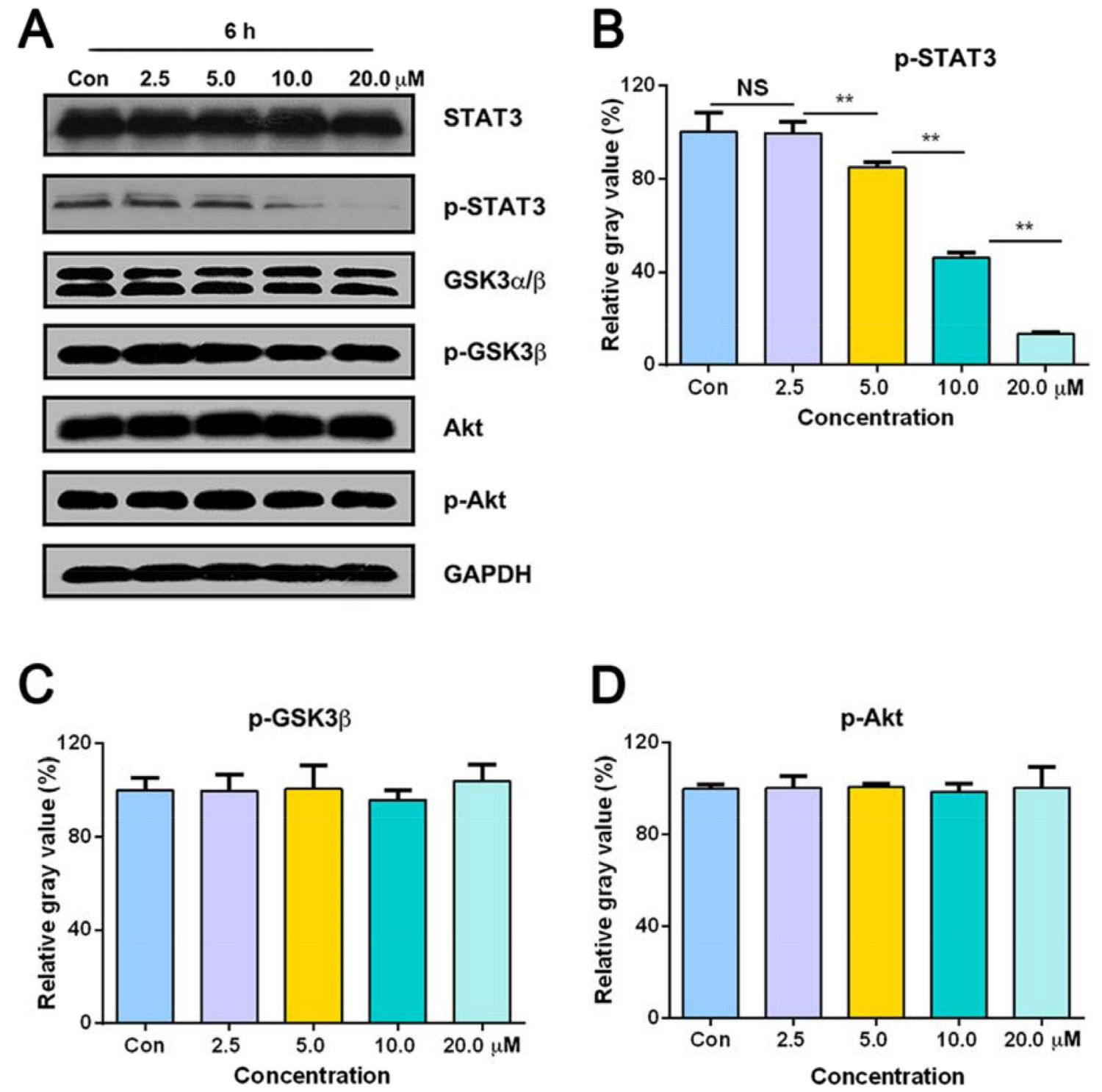

Figure 7: Total and phosphorylated levels of STAT3, GSK3 $\alpha / \beta$ and Akt after treatment with $0,2.5,5.0,10.0,20.0 \mu M$ parthenolide for 6 h. (A) Expression of STAT3, p-STAT3, GSK3 $\alpha / \beta$, p-GSK3 $\beta$, Akt and p-Akt in GLC-82 cells. (B, C, D) Gray intensity analysis of Western blot results of p-STAT3, p-GSK3 $\beta$ and p-Akt. NS $=$ no significance, ${ }^{*} p<0.05,{ }^{* *} p<0.01, n \geq 3$. 
CA) were purchased from Thermo Fisher Scientific Inc.. Fetal bovine serum (FBS) were bought from Zhejiang Tianhang Biotechnology Co.,Ltd. (Hangzhou, China). 3-(4,5-dimethylthiazolyl-2)-2,5-diphenyl tetrazolium bromide (MTT) was obtained from MP Biomedicals Inc.. PrimeScript ${ }^{\mathrm{T}}$ RT Master Mix and SYBR ${ }^{\circledR}$ Premix Ex Taq ${ }^{\mathrm{TM}}$ (Tli RNaseH Plus) were purchased from Takara Bio Inc. (Dalian, China). The following antibodies were used: anti-Erk 1/2, anti-p-Erk 1/2, anti-MEK, anti-p-MEK, anti-GSK3 $\alpha / \beta$, anti-GSK3 $\beta$, anti-c-Myc, anti-Akt, antiAkt (Ser 473), anti-STAT3, anti-p-STAT3 (Cell Signaling Technology, Inc.), anti-B-Raf (Abcam Inc., Cambridge, UK), anti-GAPDH (Bioworld Technology Inc., St Louis Park, MN). Primers for B-Raf, c-Myc and GAPDH were synthesized by Invitrogen Inc.(Carlsbad, CA).

\section{Cell culture}

GLC-82, A549 cell lines were kindly provided by Prof. Li-wu Fu (Sun Yat-sen University, Guangzhou, China); H1650, H1299, PC-9 cells were presented by Prof. Zhi Shi (Jinan University, Guangzhou, China). GLC82, A549 cells were cultured in RMPI 1640 medium and H1650, H1299, PC-9 cells were maintained in DMEM medium at $37^{\circ} \mathrm{C}$ in a $5 \% \mathrm{CO}_{2}$ incubator. Both mediums were supplemented with $10 \%$ FBS and $100 \mathrm{U} / \mathrm{mL}$ each of penicillin and streptomycin [32].

\section{Human tissue preparation}

Human lung tumor tissues were collected from 50 patients between 2011 and 2014 at Shanxi Provincial People's Hospital. The patients (males, $n=34$; females, $n=16$ ) ranged from 42 to 81 years of age and accepted no chemotherapy before operation. The tissue samples were immediately fixed in 4\% paraformaldehyde in PBS and was embedded in paraffin for immunohistochemistry. All participants provided written informed consent. The study conformed to the ethical guidelines and was approved by the hospital Ethics Committee [33].

\section{MTT assay}

The effects of parthenolide on different NSCLC cells viability were estimated using MTT. All the five cell lines were respectively seeded in 96-well plates for $24 \mathrm{~h}$ and treated with parthenolide with gradient concentration for $72 \mathrm{~h}$. MTT solution was then added for 4h. Absorbance at 540/655 $\mathrm{nm}$ was recorded using Epoch Microplate Spectrophotometer (BioTek Instruments, Inc.). Cell survival was calculated with the following formula: survival $(\%)=($ mean experimental absorbance/mean control absorbance) $\times 100 \%$ [34].

\section{Scratch wound healing assay}

For scratch experiment, GLC-82 cells were seeded on six-well plates and cells were allowed to reach confluence overnight. Subsequently, a $200 \mu \mathrm{L}$ pipette tip was used to scratch the cell monolayer and the wounded cell layer was washed to remove loose cells. Medium containing graded of parthenolide were added to the plates. After 0, 24 and $48 \mathrm{~h}$ culture, images were captured. Cell motility was determined according to the percentage of the repaired area [35].

\section{Clone formation assay}

GLC-82 cells were seeded into six-well plates at a density of 2000 cells per well. After treatment with parthenolide for indicated time, cells were washed with PBS, fixed in methanol for $15 \mathrm{~min}$, and stained with $0.5 \%$ crystal violet for $15 \mathrm{~min}$. Visualized colonies were then photographed [36].

\section{Annexin V-FITC/PI apoptosis detection}

Apoptosis rates were quantified by Annexin V-FITC/PI apoptosis detection kit (KeyGEN, Nanjing, China) according to the manufacturer's protocol. Briefly, after cells were treated with indicated concentrations of parthenolide for $72 \mathrm{~h}$, cells were collected and washed twice with ice-cold PBS. Then $5 \times 10^{5}$ cells were resuspended in $0.5 \mathrm{~mL}$ binding buffer containing Annexin-V (1:50) and $40 \mathrm{ng} /$ sample of PI for $30 \mathrm{~min}$ at $37^{\circ} \mathrm{C}$ in the dark. Subsequently, the cells were determined by flow cytometer (Becton Dickinson, NY) and analyzed by CellQuest software. At least 10,000 cells were analyzed for each sample. The apoptosis rate $(\%)=$ (the number of apoptotic cells/the number of total cells observed) $\times 100 \%$ [37].

\section{Western blotting analysis}

Cultured cells were collected in lysis buffer (Cell Signalling Technology, Danvers, MA). Equal amounts of proteins were separated on $10-12 \%$ sodium dodecyl sulfatepolyacrylamide gel electrophoresis (SDS-PAGE) and transferred onto PVDF membranes (Millipore, Boston, MA). Membranes were blocked with 5\% non-fat milk, incubated with primary antibodies at $4^{\circ} \mathrm{C}$ overnight. After incubation with horseradish peroxidase (HRP) conjugated secondary antibodies for $2 \mathrm{~h}$, blots were revealed by enhanced chemiluminescence procedures according to the manufacturer's protocol [38].

\section{RNA interference and infection}

SiRNA sequences targeting B-Raf, c-Myc and a non-targeting control were purchased from Cell Signaling Technology, Inc. and Santa Cruz Biotechnology, Inc. (Santa Cruz, CA). Transfection was performed according to the manufacturer's instructions. GLC-82 cells were seeded in six-well plates and transfected transiently with siRNA using Lipofectamine ${ }^{\circledR} 2000$ (Invitrogen, Carlsbad, 
CA). After transfection for $24 \mathrm{~h}, \mathrm{GLC}-82$ cells were used for Western blot and RT-QPCR [39].

\section{Real-time quantitative reverse transcription-PCR}

Total RNA was extracted from cells using TRIzol ${ }^{\circledR}$ Reagent (Ambio, CA), and 500 ng RNA per 10 ul reaction solution was used to synthesize cDNA using the PrimeScript ${ }^{\mathrm{TM}}$ RT Master Mix (Takara, Japan). cDNA was amplified and quantified by RT-QPCR with SYBR ${ }^{\circledR}$ Premix Ex Taq ${ }^{\mathrm{TM}}$ (Tli RNaseH Plus) (Takara, Japan). GAPDH was used as the internal control. The primer sequences for the PCR amplification of B-Raf, c-Myc and GAPDH were: B-Raf primer set (forward, 5'-AGA AAG CAC TGA TGA TGA GAG G-3'; reverse, 5'-GGA AAT ATC AGT GTC CCA ACC A-3'), c-Myc primer set (forward, 5'-GCT CAT TTC TGA AGA GGA CTT GT-3'; reverse, 5'-AGG CAG TTT ACA TTA TGG CTAAAT C-3'), and GAPDH primer set (forward, 5'-GGA AGG TGA AGG TCG GAG TCA-3'; reverse, 5'GTC ATT GAT GGC AAC AAT ATC CAC T-3') [40].

\section{Immunohistochemistry}

Paraffin-embedded tumor tissues were sectioned and deparaffinized with xylene. The slides were immersed into different concentrations of alcohol for rehydration and then in $3 \% \mathrm{H}_{2} \mathrm{O}_{2}$ to block endogenous peroxidase. After washing with distilled water, the slides were incubated with different grades of antibody. Immunoreactions were visualized using DAB substrate, counterstained by hematoxylin and coverslipped for microscopic examination [41].

\section{Statistical analysis}

Each experiment was repeated at least three times. All numerical data were presented as mean \pm standard deviation. Statistical difference in each assay was analyzed by Graphpad Prism 6, and was tested for significance using $t$ test and ANOVA analysis of variance. $P<0.05$ was considered significant.

\section{CONCLUSIONS}

In conclusion, parthenolide exhibited potent cytotoxicity towards human NSCLC cells. Parthenolide could target on B-Raf and inhibited the MAPK/Erk pathway signaling which is independent of PI3K pathway. Inhibition of STAT3 phosphorylation was also found to be related with the potent cytotoxicity of parthenolide. The involved mechanism was summarized in Figure 8.

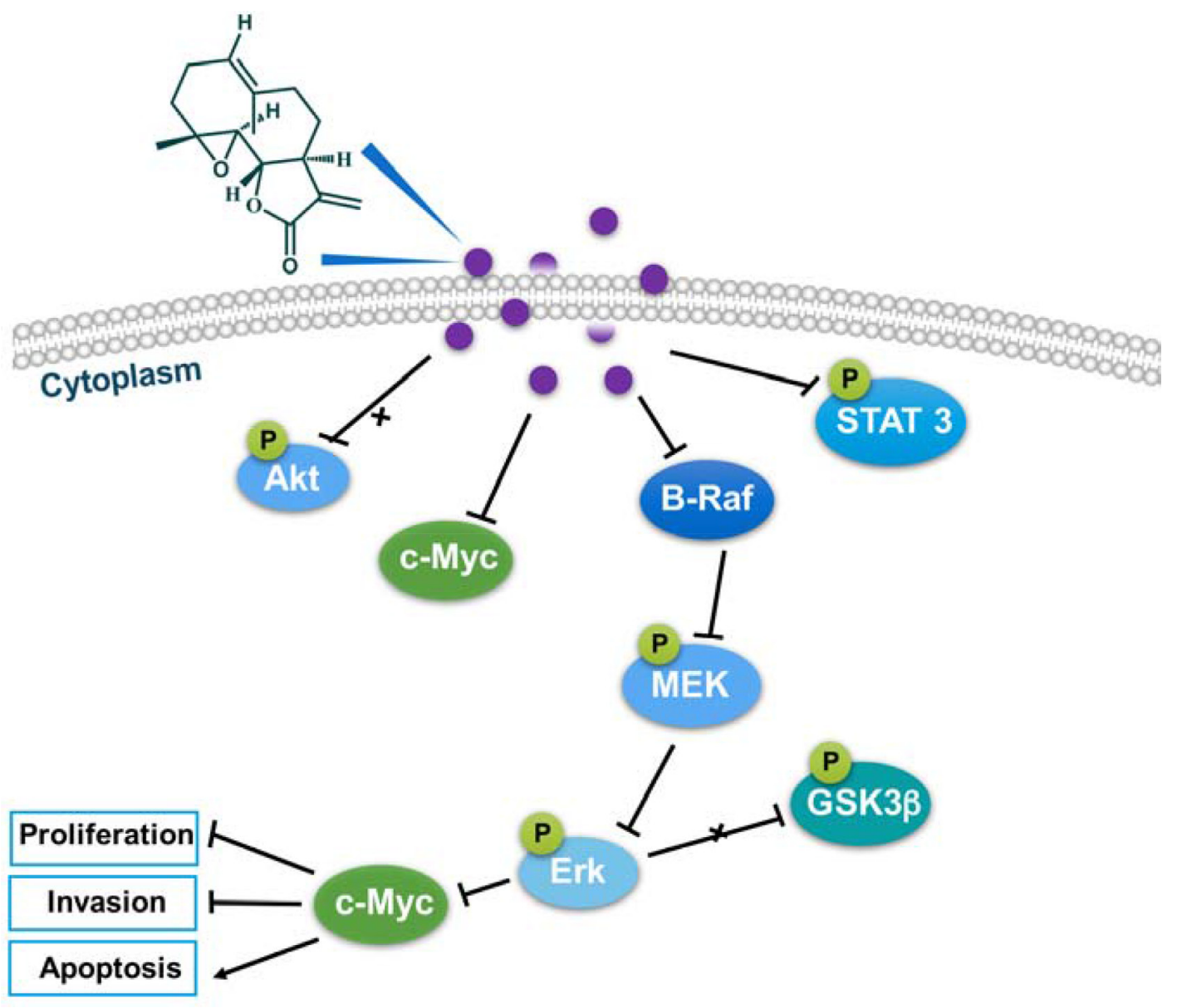

Figure 8: The involved mechanism of parthenolide against NSCLC cells. 


\section{ACKNOWLEDGMENTS}

We thank the State Key Laboratory of Sun Yan-Sen Memorial Hospital for providing instrument for apoptosis detection.

\section{CONFLICTS OF INTEREST}

The authors declare no competing financial interest.

\section{FUNDING}

The work was supported by National Natural Science Foundation of China (81473320), the Fund from Guangdong Science and Technology Department (2016A020226024), Fund of Guangdong Education Department (2015KTSCX112), the Fund of Guangzhou Science and Technology Program (201605100911458), Science Fund of the Education Bureau of Guangzhou (1201410039) (to J.Y. Zhang), the National Science Foundation for Young Scientists of Shanxi Province (2014021037-6) (to Y.Y. Yan), Science and Technology Research Project of Shanxi Province (20140313011-2) (to H. Bi).

\section{Authors' contributions}

M.T. Lin, Y.Y. Yan and H. Bi contributed equally to this paper and performed the experiments. W.J. Huang, L.L. Zhang and J.X. Ma wrote the paper. G.P. Zhang, G.S. Zhang, S.L. Tang and Y. Liu reviewed and edited the manuscript. J.Y. Zhang conceived and designed the study. All authors read and approved the manuscript.

\section{REFERENCES}

1. World Health Organization. Global health observatory data repository. 2011; Available from: http://apps.who.int/gho/ data/node.main.CODWORLD?lang=en

2. Ferlay J, Soerjomataram I, Dikshit R, Eser S, Mathers C, Rebelo M, Parkin DM, Forman D, Bray F. Cancer incidence and mortality worldwide: sources, methods and major patterns in GLOBOCAN 2012. Int J Cancer. 2015; 36:359-386.

3. Wu H, Zhou J, Zeng C, Wu D, Mu Z, Chen B, Xie Y, Ye Y, Liu J. Curcumin increases exosomal TCF21 thus suppressing exosome-induced lung cancer. Oncotarget. 2016; 7:87081-87090. doi: 10.18632/oncotarget.13499.

4. Mountain CF. The international system for staging lung cancer. Semin Surg Oncol. 2000; 18:106-115.

5. Hsu HC, Thiam TK, Lu YJ, Yeh CY, Tsai WS, You JF, Hung HY, Tsai CN, Hsu A, Chen HC, Chen SJ, Yang TS. Mutations of KRAS/NRAS/BRAF predict cetuximab resistance in metastatic colorectal cancer patients. Oncotarget. 2016; 7:22257-22270. doi: 10.18632/oncotarget.8076.

6. Califano R, Abidin A, Tariq NU, Economopoulou P, Metro G, Mountzios G. Beyond EGFR and ALK inhibition: unravelling and exploiting novel genetic alterations in advanced non small-cell lung cancer. Cancer Treat Rev. 2015; 41:401-411.

7. Mok TS, Wu YL, Thongprasert S, Yang $\mathrm{CH}$, Chu DT, Saijo N, Sunpaweravong P, Han B, Margono B, Ichinose Y, Nishiwaki Y, Ohe Y, Yang JJ, et al. Gefitinib or carboplatinpaclitaxel in pulmonary adenocarcinoma. N Engl J Med. 2009; 361:947-957.

8. Cragg GM, Grothaus PG, Newman DJ. Impact of natural products on developing new anti-cancer agents. Chem Rev. 2009; 109:3012-3043.

9. Kingston DG. Modern natural products drug discovery and its relevance to biodiversity conservation. J Nat Prod. 2011; $74: 496-511$.

10. Akram Ghantous, Ansam Sinjab, Zdenko Herceg, Nadine Darwiche. Parthenolide: from plant shoots to cancer roots. Drug Discov Today. 2013; 18:894-905.

11. Ikawa S, Fukui M, Ueyama Y, Tamaoki N, Yamamoto T, Toyoshima K. B-raf, a new member of the raf family, is activated by DNA rearrangement. Mol Cell Biol. 1988; 8:2651-2654.

12. Wellbrock C, Karasarides M, Marais R. The RAF proteins take centre stage. Nat Rev Mol Cell Biol. 2004; 5:875-885.

13. Davies H, Bignell GR, Cox C, Stephens P, Edkins S, Clegg S, Teague J, Woffendin H, Garnett MJ, Bottomley W, Davis N, Dicks E, Ewing R, et al. Mutations of the BRAF gene in human cancer. Nature. 2002; 417:949-954.

14. Pollock PM, Meltzer PS. A genome-based strategy uncovers frequent BRAF mutations in melanoma. Cancer Cell. 2002; 2:5-7.

15. Pollock PM, Harper UL, Hansen KS, Yudt LM, Stark M, Robbins CM, Moses TY, Hostetter G, Wagner U, Kakareka J, Salem G, Pohida T, Heenan P, et al. High frequency of BRAF mutations in nevi. Nat Genet. 2003; 33:19-20.

16. Yuen ST, Davies H, Chan TL, Ho JW, Bignell GR, Cox C, Stephens P, Edkins S, Tsui WW, Chan AS, Futreal PA, Stratton MR, Wooster R, et al. Similarity of the phenotypic patterns associated with BRAF and KRAS mutations in colorectal neoplasia. Cancer Res. 2002; 62:6451-6455.

17. Marchetti A, Felicioni L, Malatesta S, Grazia Sciarrotta M, Guetti L, Chella A, Viola P, Pullara C, Mucilli F, Buttitta F. Clinical features and outcome of patients with non-smallcell lung cancer harboring BRAF mutations. J Clin Oncol. 2011; 29:3574-3579.

18. Sanchez-Torres JM, Viteri S, Molina MA, Rosell R. BRAF mutant non-small cell lung cancer and treatment with BRAF inhibitors. Transl Lung Cancer Res. 2013; 2:244-250.

19. Paik PK, Arcila ME, Fara M, Sima CS, Miller VA, Kris MG, Ladanyi M, Riely GJ. Clinical characteristics of patients with lung adenocarcinomas harboring BRAF mutations. J Clin Oncol. 2011; 29:2046-2051.

20. Zhang D, Qiu L, Jin X, Guo Z, Guo C. Nuclear factorkappaB inhibition by parthenolide potentiates the efficacy 
of Taxol in non-small cell lung cancer in vitro and in vivo. Mol Cancer Res. 2009; 7:1139-1149.

21. Hauschild A, Grob JJ, Demidov LV, Jouary T, Gutzmer R, Millward M, Rutkowski P, Blank CU, Miller WH, Jr, Kaempgen E, Martin-Algarra S, Karaszewska B, et al. Dabrafenib in BRAF-mutated metastatic melanoma: a multicentre, open-label, phase 3 randomised controlled trial. Lancet. 2012; 380:358-365.

22. Cantwell-Dorris ER, O'Leary JJ, Sheils OM. BRAFV600E: implications for carcinogenesis and molecular therapy. Mol Cancer Ther. 2011; 10:385-394.

23. Papin C, Denouel-Galy A, Laugier D, Calothy G, Eychene A. Modulation of kinase activity and oncogenic properties by alternative splicing reveals a novel regulatory mechanism for B-Raf. J Biol Chem. 1998; 273:24939-24947.

24. Gao ZW, Zhang DL, Guo CB. Paclitaxel efficacy is increased by parthenolide via nuclear factor-kappaB pathways in in vitro and in vivo human non-small cell lung cancer models. Curr Cancer Drug Targets. 2010; 10:705-715.

25. Chapman PB, Hauschild A, Robert C, Haanen JB, Ascierto P, Larkin J, Dummer R, Garbe C, Testori A, Maio M, Hogg D, Lorigan P, Lebbe C, et al. Improved survival with vemurafenib in melanoma with BRAF V600E mutation. N Engl J Med. 2011; 364:2507-2516.

26. Pullikuth AK, Catling AD. Scaffold mediated regulation of MAPK signaling and cytoskeletal dynamics: a perspective. Cell Signal. 2007; 19:1621-1632.

27. Yang SH, Sharrocks AD, Whitmarsh AJ. MAP kinase signalling cascades and transcriptional regulation. Gene. 2013; 513:1-13.

28. Davis RJ. Transcriptional regulation by MAP kinases. Mol Reprod Dev. 1995; 42:459-467.

29. Meyer T, Vinkemeier U. STAT nuclear translocation: potential for pharmacological intervention. Expert Opin Ther Targets. 2007; 11:1355-1365.

30. Sobota R, Szwed M, Kasza A, Bugno M, Kordula T. Parthenolide inhibits activation of signal transducers and activators of transcription (STATs) induced by cytokines of the IL-6 family. Biochem Biophys Res Commun. 2000; 267:329-333.

31. De Luca A, Maiello MR, D'Alessio A, Pergameno M, Normanno N. The RAS/ RAF/ MEK/ ERK and the PI3K/ AKT signalling pathways: role in cancer pathogenesis and implications for therapeutic approaches. Expert Opin Ther Targets. 2012; 16:S17-27.
32. Lin M, Tang S, Zhang C, Chen H, Huang W, Liu Y, Zhang J. Euphorbia factor L2 induces apoptosis in A549 cells through the mitochondrial pathway. Acta Pharm Sin B. 2017; 7:59-64.

33. Zuckerman V, Sokolov E, Swet JH, Ahrens WA, Showlater V, Iannitti DA, Mckillop IH. Expression and function of lysophosphatidic acid receptors (LPARs) 1 and 3 in human hepatic cancer progenitor cells. Oncotarget. 2016; 7: 2951-2967. doi: 10.18632/oncotarget.6696.

34. Zhang JY, Yi T, Liu J, Zhao ZZ, Chen HB. Quercetin induces apoptosis via the mitochondrial pathway in $\mathrm{KB}$ and KBv200 cells. J Agric Food Chem. 2013; 61:2188-2195.

35. Cao H, Huang Y, Wang L, Wang H, Pang X, Li K, Dang W, Tang H, Wei L, Su M, Tang C, Chen T. Leptin promotes migration and invasion of breast cancer cells by stimulating IL-8 production in M2 macrophages. Oncotarget. 2016; 7:65441-65453. doi: 10.18632/oncotarget.11761.

36. Wu XY, Liu WT, Wu ZF, Chen C, Liu JY, Wu GN, Yao XQ, Liu FK, Li G. Identification of HRAS as cancer-promoting gene in gastric carcinoma cell aggressiveness. Am J Cancer Res. 2016; 6:1935-1948.

37. Zhang JY, Lin MT, Tung HY, Tang SL, Yi T, Zhang YZ, Tang YN, Zhao ZZ, Chen HB. Bruceine D induces apoptosis in human chronic myeloid leukemia K562 cells via mitochondrial pathway. Am J Cancer Res. 2016; 6:819-826.

38. Tao YW, Lin YC, She ZG, Lin MT, Chen PX, Zhang JY. Anticancer activity and mechanism investigation of beauvericin isolated from secondary metabolites of the mangrove endophytic fungi. Anticancer Agents Med Chem. 2015; 15:258-266.

39. Zhang JY, Tao LY, Liang YJ, Yan YY, Dai CL, Xia XK, She ZG, Lin YC, Fu LW. Secalonic acid D induced leukemia cell apoptosis and cell cycle arrest of $\mathrm{G}(1)$ with involvement of GSK-3beta/beta-catenin/c-Myc pathway. Cell Cycle. 2009; 8:2444-2450.

40. Park S, Cheng SL, Cui JY. Characterizing drugmetabolizing enzymes and transporters that are bona fide CAR-target genes in mouse intestine. Acta Pharm Sin B. 2016; 6:475-491.

41. Chen K, Cheng G, Zhang F, Zhang N, Li D, Jin J, Wu J, Ying L, Mao W, Su D. Prognostic significance of programmed death-1 and programmed death-ligand 1 expression in patients with esophageal squamous cell carcinoma. Oncotarget. 2016; 7:30772-30780. doi: 10.18632/oncotarget.8956. 